\title{
Novel Computerized Method for Measurement of Retinal Vessel Diameters
}

\author{
Hichem Guedri ${ }^{1, *}$, Mariem Ben Abdallah ${ }^{1}$, Fraj Echouchene ${ }^{1}$ and Hafedh Belmabrouk ${ }^{1,2}$ \\ 1 Electronics and Microelectronics Laboratory, Physics Department, Faculty of Sciences, Monastir University, \\ Monastir 5019, Tunisia; mariembenabdallah3@gmail.com (M.B.A.); frchouchene@yahoo.fr (F.E.); \\ Hafedh.Belmabrouk@fsm.rnu.tn (H.B.) \\ 2 Department of Physics, College of Science AlZulfi, Majmaah University, Majmaah 15341, Saudi Arabia \\ * Correspondence: himougu@yahoo.fr; Tel.: +21-697-456-167
}

Academic Editor: Shaker A. Mousa

Received: 8 February 2017; Accepted: 17 March 2017; Published: 27 March 2017

\begin{abstract}
Several clinical studies reveal the relationship between alterations in the topologies of the human retinal blood vessel, the outcrop and the disease evolution, such as diabetic retinopathy, hypertensive retinopathy, and macular degeneration. Indeed, the detection of these vascular changes always has gaps. In addition, the manual steps are slow, which may be subjected to a bias of the perceiver. However, we can overcome these troubles using computer algorithms that are quicker and more accurate. This paper presents and investigates a novel method for measuring the blood vessel diameter in the retinal image. The proposed method is based on a thresholding segmentation and thinning step, followed by the characteristic point determination step by the Douglas-Peucker algorithm. Thereafter, it uses the active contours to detect vessel contour. Finally, Heron's Formula is applied to assure the calculation of vessel diameter. The obtained results for six sample images showed that the proposed method generated less errors compared to other techniques, which confirms the high performance of the proposed method.
\end{abstract}

Keywords: active contour; blood vessel diameter; Douglas-Peucker algorithm; eye diseases; retinal image

\section{Introduction}

Medical imaging encompasses methods for imaging human organs; this advanced technology makes it possible to improve the diagnosis and effective treatments of these organs [1-4]. Furthermore, the use of precise diagnosis and monitoring instruments can more quickly take into account and review various medical conditions and health events in clinical examinations $[5,6]$.

Blood vessels play a pivotal role in the discovery, the observation, and the diagnosis of some medical conditions [7-10]. For example, retinal vessels may be affected by certain diseases such as diabetic retinopathy, hypertensive retinopathy, and macular degeneration $[7,8]$. They affect the structural characteristics of the blood vessels of the human retina. Therefore, the study and the analysis of the vessel geometric characteristics such as vessel diameter, branch angles, and branch lengths have become the basis of medical applications related to early diagnosis and effective monitoring of these diseases.

Therefore, this paper will seek to explore and identify a tool to measure the blood vessel diameter in retinal images and to acquire a computerized system that can automatically predict the change in the vessels width.

There are many methods used to detect blood vessel diameters. Kumar et al. [11] have introduced an automatic method for measuring the vessel diameter; this method is based on the Linear Discriminate Analysis (LDA). Bhuiyan et al. [12] have proposed a technique for measuring 
blood vessel width; this method is based on the vessel edge and centerline, in addition to the image gradient segmentation technique (ARG) for the vessel edge's detection. Furthermore, it is based on the unsupervised texture classification method for obtaining the vessel centerline. At the final step, a mask rotation invariant was applied for center-line pixels, whose center corresponds to the pixels' position. The potential pixels were drawn from the edge image using a continuous increment from lower to higher distance and orientation. El Abbadi et al. [13] have suggested a strategy to identify the retinal blood vessel diameter based on mask creation and specifically to measure the blood vessel diameter. Gao et al. [14,15] have proposed another method to measure the blood vessel diameter, which incorporates the use of a tracking technique. Twin Gaussian functions are introduced for modeling the distribution of a gray-level profile over a vessel cross section. The tracking technique is used to study the variations of vessel diameter in the direction of the vessel longitude axis. In addition, Tison et al. [16] have introduced a method to estimate the blood vessel diameter using active contours. The system proposed consists of a segmentation procedure which uses two active contours to detect the blood vessel outline and an approach to measure blood vessel diameters as the distance between two points of the edge. Lowell et al. [17] have proposed an algorithm to measure the vessel diameter to subpixel accuracy. This method is based on a two-dimensional difference of Gaussian model, where the model is optimized to fit a two-dimensional intensity vessel segment. Our proposed algorithm improves the performance of the measurement results compared with the works described above.

This paper is organized as follows: Section 2 illustrates the methodology of the proposed problem that has driven our research. Next, we briefly describe the datasets and the principal characteristics of the retinal image. Thereafter, we describe the method to convert the image from the space gray to binary image in order to introduce a thinning algorithm to get a 1-pixel-wide skeleton representing the center line of the vessel tree. The next step consists of a pixel classification algorithm which is dedicated to extracting the pixel information. We also try to explore the essentials of the Douglas-Peucker method used to determine the characteristic points. Afterward, we describe the detection of the contour using the snake method and a brief survey of his existing equations. Then, we illustrate the implementation of the proposed algorithm. The experiments applied on retinal images are explained step by step and the results are presented in Section 3. In Section 4, we discuss the properties of the obtained results. Finally, Section 5 concludes the paper by summarizing the proposed method.

\section{Material and Methods}

\subsection{Proposed Method}

The most common diseases of the eye and its related structures are diabetic retinopathy, hypertensive retinopathy, and macular degeneration. Diabetic retinopathy is a disease that happens due to damage in the retina blood vessels for people who have type 1 or 2 diabetes [1-7]. In addition, high blood pressure can induce harm to retina blood vessels and they can become narrow, limiting the function of the retina, and putting pressure on the optic nerve, causing vision problems. Macular degeneration occurs when abnormal blood vessels develop under the retina and macula; the condition can make blood vessels bleed or leak fluid. We can notice that these diseases have a direct consequence on the regional anatomy of the human retinal blood vessel. In this research study, we are interested in developing an automatic diagnosis system of the changes in levels of the human retina topology that can help the ophthalmologist to diagnose the patient [11-22].

The aim of this paper is to measure the diameter of blood vessels in retinal images. Figure 1 illustrates the different steps of our approach. 


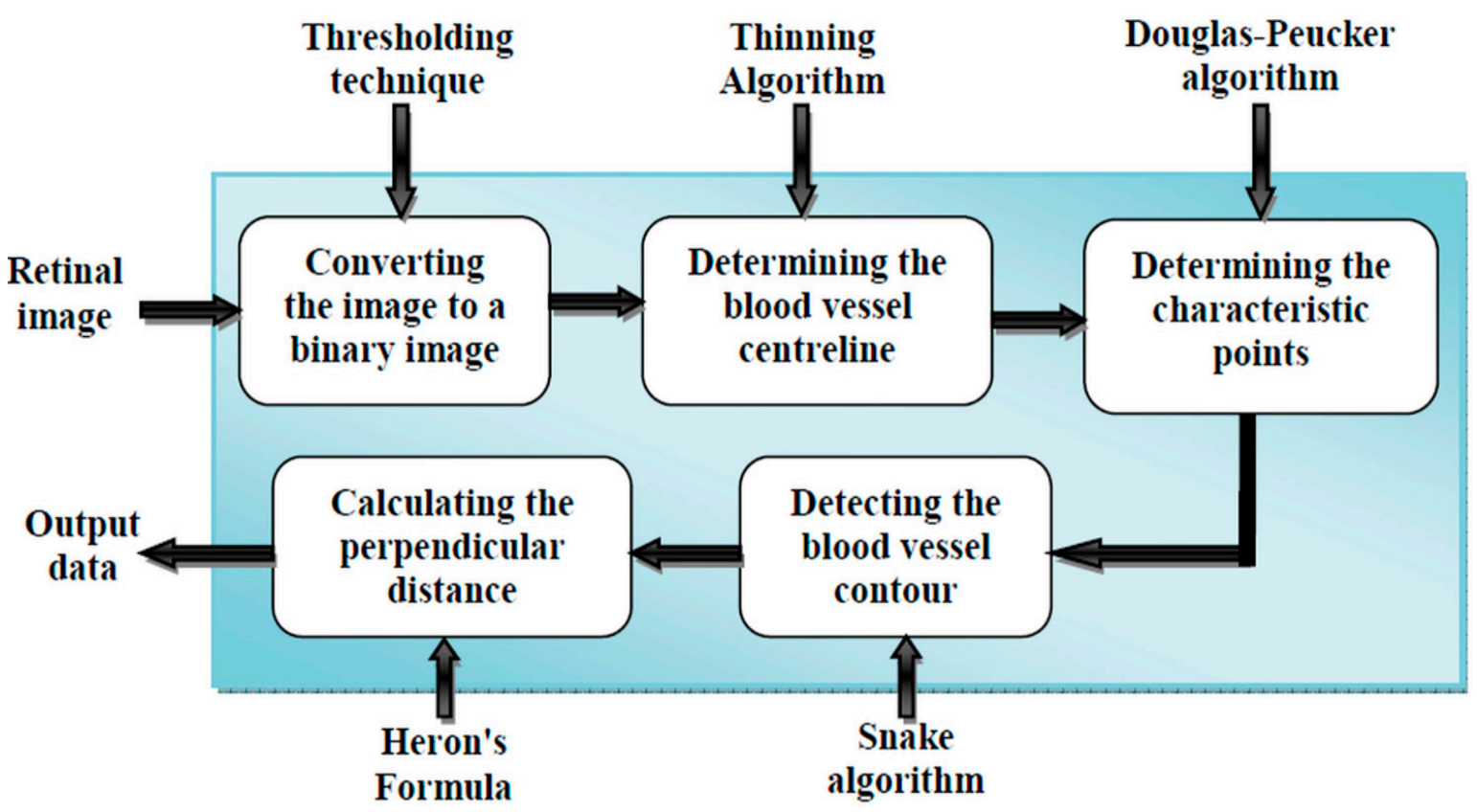

Figure 1. Block diagram of the proposed method.

(i) First, we will give the image source and introduce its features.

(ii) Then, we will present pre-processing of the 2D image.

(iii) Subsequently, the determination of the control point using the Douglas-Peucker algorithm will be explained.

(iv) Then, we will detect the blood vessel contour using the active contour technique.

(v) Finally, the Heron's formula will be presented to determine the blood vessels diameter.

\subsection{Retinal Image Segmentation}

\subsubsection{Image Source}

There are several retinal images databases available. For example, one of the most used databases for vessel segmentation is the STARE (STructured Analysis of the Retina) database [23], which contains low-resolution images. On the other hand, there are other databases with higher resolution such as High-Resolution Fundus (HRF) Image Database [24].

\section{STARE Database}

In 1975, Michael Goldbaum, M.D. created and initialized the STARE Project, at the University of California, San Diego [23]. This project provides a full set of 400 retinal raw clinical images. Four sets of images are made usable, one set of 20 raw images was captured by a Topcon TRV-50 fundus camera with a 35 degree field of view; the slides that were produced were digitized in order to produce images with $700 \times 605$ pixel resolution with 24-bits per pixel (Figure 2a). The two other sets of 20 images (set 2 and 3) come with an additional vessel segmentation that was hand labeled-the first set was provided by Adam Hoover (Figure $2 b$ ) and the second set of images was provided by Valentina Kouznetsova (Figure 2c). The fourth set provides sample results as produced by the matched spatial filter probing algorithm (Figure 2d) [25]. 


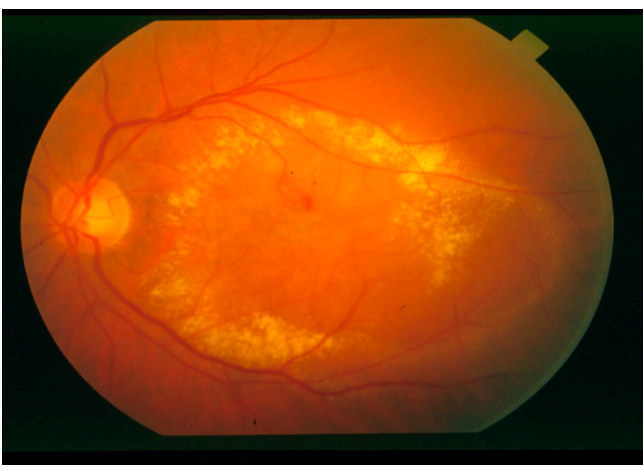

(a)

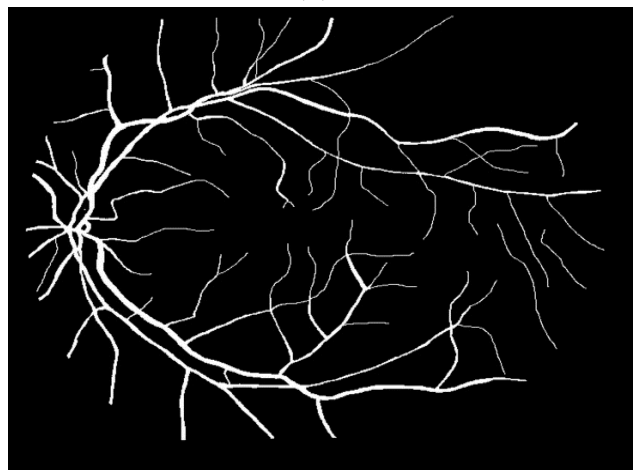

(c)

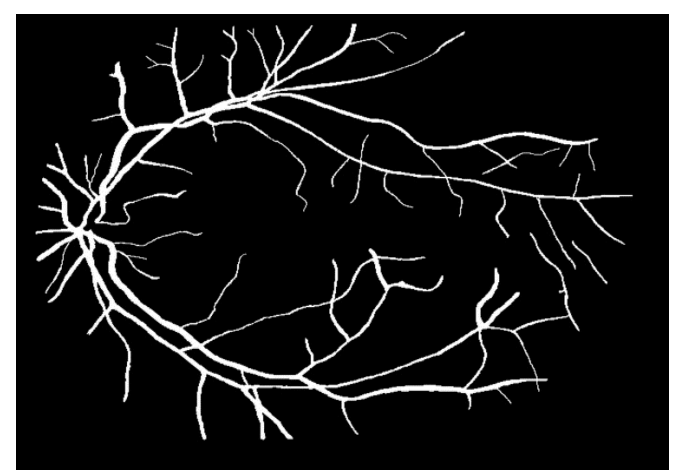

(b)

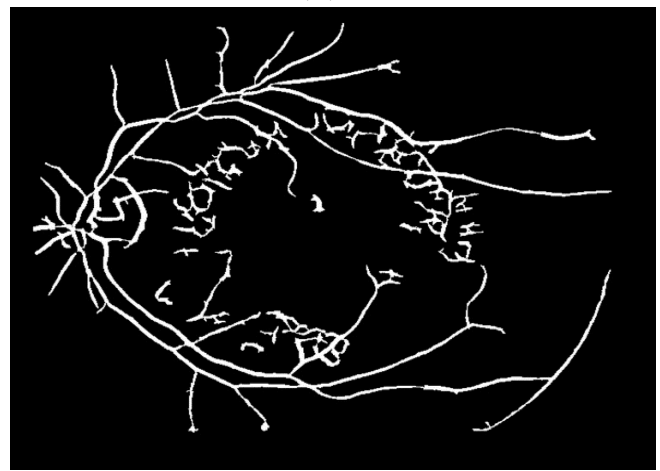

(d)

Figure 2. Example of a fundus image used: (a) Example of a raw image im0002.jpg; (b) Vessel networks marked by hand provided by Adam Hoover; (c) Vessel networks marked by hand provided by Valentina Kuznetsova; (d) Vessel networks produced by matched spatial filter probing algorithm.

High-Resolution Fundus (HRF) Image Database

This database contains 45 fundus images. These images are divided into three sets: 15 images of healthy patients, 15 images of patients with diabetic retinopathy, and 15 images of glaucomatous patients [24]. All of these images were taken using a CANON CF-60UVi camera, the image's dimensions for the JPEG still images shooting are $3504 \times 2336$ pixels. Also, manual labeling of the vessels was done by experts in vessel segmentation and binary vessel segmentation images are available for each image. Figure 3a shows an example of a fundus image from the High-Resolution Fundus (HRF) Image Database with the corresponding manual segmentation (Figure 3b).

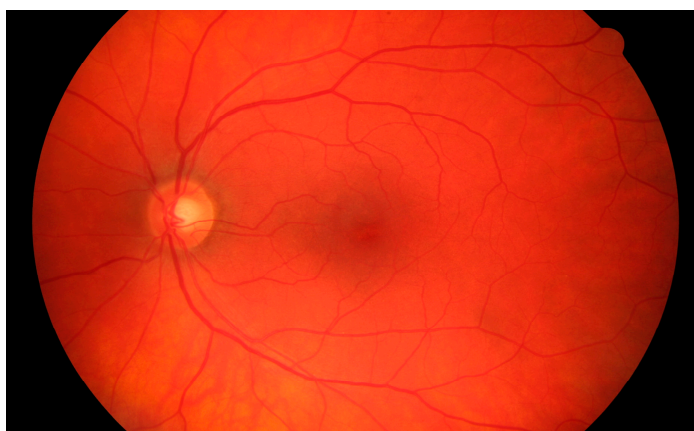

(a)

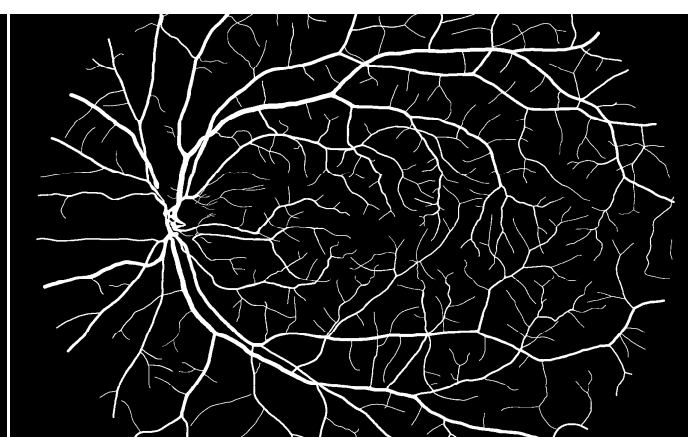

(b)

Figure 3. Example of a fundus image from the proposed database: (a) Example of a raw image im0002.jpg; (b) The manual segmentation of the vessels. 


\subsubsection{Binary and Skeletonization Image}

The next step of our work is to convert pictures into a binary image [26-29]. The binarization process converts a pixel code to multiple bits of 4,8 , or more on a single bit. The most used method is the thresholding technique $[30,31]$ :

$$
I_{B}(i, j)=\left\{\begin{array}{l}
0 \text { if } S>I_{E}(i, j) \\
1 \text { if } S<I_{E}(i, j)
\end{array}\right.
$$

where $I_{\mathrm{E}}$ is the input image and $I_{B}$ is the binary image.

The blood vessel skeleton allows the complexity of the form to be reduced by finding the optimal curve. Thinning algorithms are typically used due to their effectiveness and reliability. The thinning algorithm is applied by removing all the points of the image contour except points that belong to the skeleton, which are left [32-34]. We can classify this algorithm as an iterative algorithm which erodes the outer pixels layers until there are no more layers that may be removed $[35,36]$.

This technique does not take into consideration the blood vessel width, but could measure other qualities such as orientation, vessel segments length, and the presence of shortcomings, in addition to endpoint and bifurcation point detections.

\subsubsection{Point Detection}

In our strategy, we apply a $3 \times 3$ sliding mask for determination of the endpoint and bifurcations points and we compute the number of the active neighboring pixels. If the center of the mask is an active pixel and the number of active neighboring pixels is 1, this point is the endpoint (Figure 4a). If there are two active neighboring pixels, this indicates an interior point (Figure $4 \mathrm{~b}$ ). Otherwise, if the number of active neighboring pixels is greater than 2 , this indicates that the point is the bifurcations point (Figure 4c) [37,38].
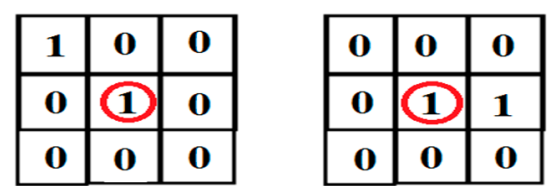

(a)
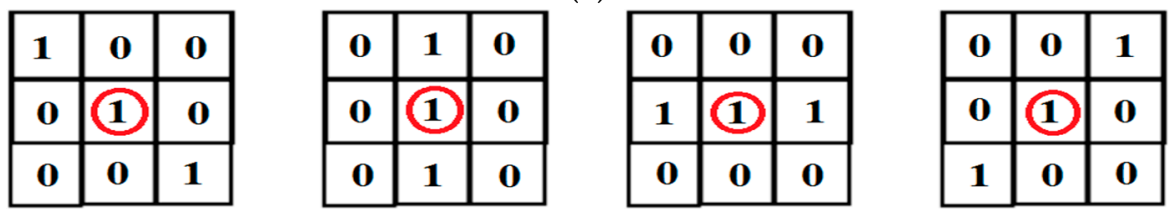

(b)

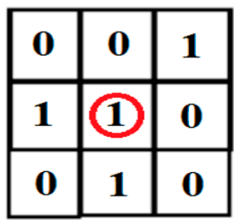

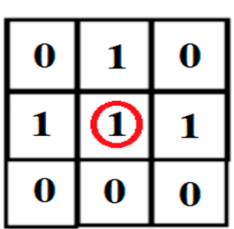

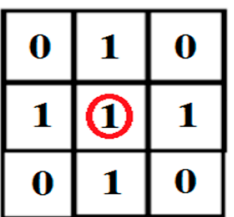

(c)

Figure 4. Pixel classification (the red circle represents the active pixels): (a) Endpoint; (b) Inner point; (c) Bifurcations point.

\subsubsection{Determination of the Characteristic Points from the Blood Vessel Curve}

The objective of the simplified polynomials algorithms is to simplify the lines by removing the extraneous bends while maintaining their overall shape and retaining the essential points of their 
shape, which are called characteristic points. There are several algorithms dedicated to accomplishing this goal, such as nth point elimination, a normal routine, and the Douglas-Peucker algorithm [39-42].

White [39] has conducted a study on these three simplified algorithms based on Marin's work [40]. He showed that the results produced by the Douglas-Peucker algorithm are the best examples of the original lines representations in $86 \%$ of all tests. Thus, this algorithm was adopted in our work.

The Douglas-Peucker algorithm

The first step of the Douglas-Peucker algorithm is to connect the end nodes of a curve with a segment P1P2 (step 1 in Figure 5). The perpendicular distance between each curve point and the segment P1P2 is measured (step 2 in Figure 5):

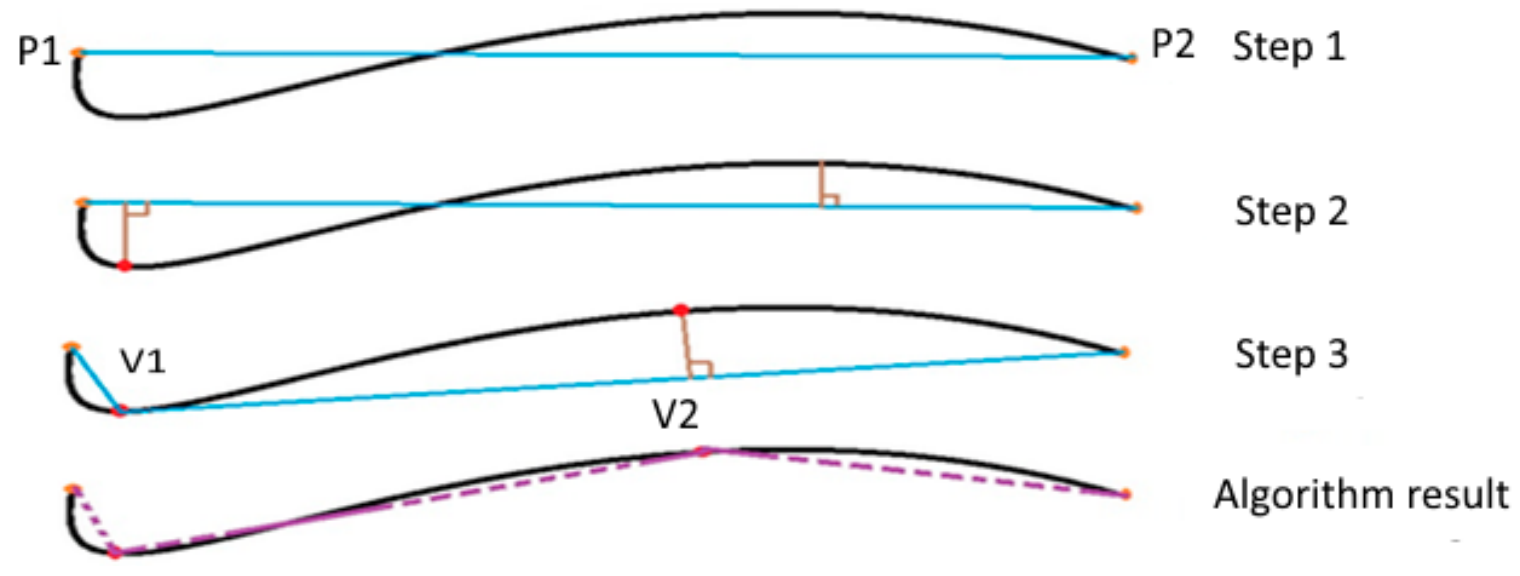

Figure 5. The different stages of the Douglas-Peucker algorithm.

If the points have a lower orthogonal distance than a defined tolerance value, then these points will be eliminated.

If not, the curve is divided by the most distant point from the P1P2 segment. This has the effect of creating two new curves V1 and V2 (step 3 in Figure 5). This process is repeated until all the distances become smaller than a tolerance value $[41,42]$.

\subsection{Edge Detection with Snake Algorithm}

The snake algorithm was proposed by Kass et al. in 1988 [43]. The idea is to move the points to bring them closer to high gradient areas while maintaining the characteristics such as the contour curvature of the point distributions or other restrictions on the order of the points [44-46]. This algorithm begins by arranging the contour evenly around the object. Subsequently, the contour has two paths: either shrink or develop (it is located inside an object at the beginning of the algorithm) and try to scan the entire object shape to have the best forms. The algorithm tries to find a better contour position in order to minimize deviations from the constraints used [43]. The algorithm stops when it is not possible to improve or simplify the implementation, when the maximum iterations number is achieved.

\subsubsection{The Snake Algorithm}

The snake model is described as a model of controlled continuity under the influence of the image forces [42]. The internal forces control the bending line characteristics, whilst the image forces serve to push the snake to detect the image characteristics. The snake position can be represented by the function $v(s)=(x(s), y(s))$ and can have its energy function written as follows:

$$
E_{\text {Snake }}^{*}=\int_{0}^{1} E_{\text {Snake }} v(s) \mathrm{dt}=\int_{0}^{1} E_{\text {int }}(v(s))+E_{\text {ext }}(v(s))
$$


where $E_{\text {int }}$ is internal energy given in equation (3), and $E_{\text {ext }}$ is the external energy given in Equation (4).

\subsubsection{The Internal Energy}

The internal energy is given in the following equation [42]:

$$
E_{\text {int }}=E_{\text {cont }}+E_{\text {curv }}=\alpha(s)\left|V_{s}(s)\right|^{2}+\beta(s)\left|V_{s s}(s)\right|^{2}
$$

where:

$E_{\text {cont }}$ is the continuity energy, it ensures that the parameterization of the points remains equidistant from each other.

$E_{\text {curv }}$ : is the curvature energy, it maintains the rigidity of the snake.

$\mathrm{v}_{\mathrm{s}}(\mathrm{s})$ : is the first derivative of screws with respect to $\mathrm{s}$.

$\mathrm{v}_{\mathrm{ss}}(\mathrm{s})$ : is the second derivative of $\mathrm{v}(\mathrm{s})$ with respect to $\mathrm{s}$.

$\alpha(\mathrm{s})$ : is the contour elasticity.

$\beta(\mathrm{s})$ : is the contour rigidity.

\subsubsection{The External Energy}

The external energy is taken to be the gradient magnitude of the image and it is given in the following equation:

$$
E_{\text {ext }}=-\gamma\left|\nabla G_{\sigma}(x, y) \times I(x, y)\right|^{2}
$$

where:

$\gamma:$ is a constant used to control the importance of the $E_{\text {ext }}$.

$\mathrm{G}_{\sigma}(\mathrm{x}, \mathrm{y})$ : is a Gaussian kernel with a scale $\sigma$.

\subsection{Methods}

\subsubsection{Initialization.}

The developed algorithm aims to support this method in three steps: The first step carried out the characteristic point detection using the Douglas-Peucker algorithm [41]. The process of this algorithm begins by plotting a line which is the linear regression for all points. This was followed by calculating the farthest point of the straight line of regression in order to calculate the new different regressions from left to right. This process will be repeated until getting an error smaller than a fixed limit. The next step will be devoted to the detection of blood vessel contour using the snake method, which is used to place an initial contour line around the shape to be detected [43]. This line will gradually deform according to the action of several forces that will pull or push it towards the shape. This dynamic is based on the notion of internal and external energy, the aim therefore being to minimize the total energy present along the curve. Finally, Heron's formula should be applied in order to detect the blood vessel diameter. This step is detailed in the next section.

\subsubsection{Determination of Vessel Diameters}

The Douglas-Peucker algorithm aims to determine the characteristic points, we then use the snake method to detect the blood vessels contour. These characteristic and contour points are used to calculate the blood vessel diameters. The computation process of the proposed algorithm is shown in Figure 6. 


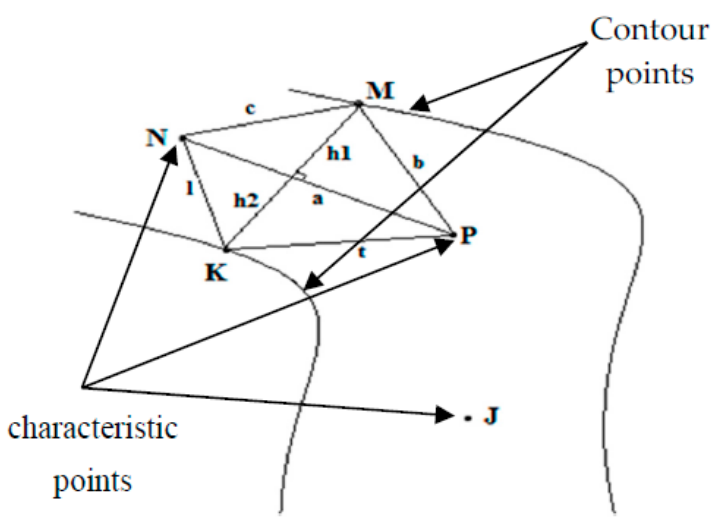

(a)

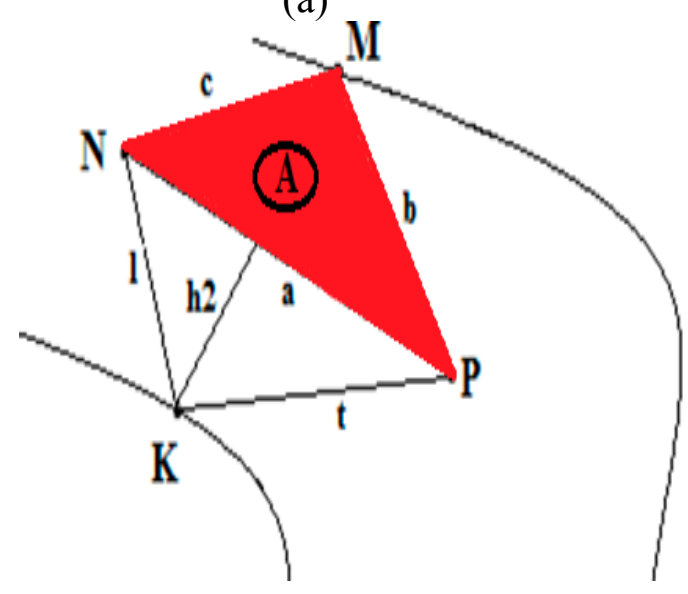

(c)

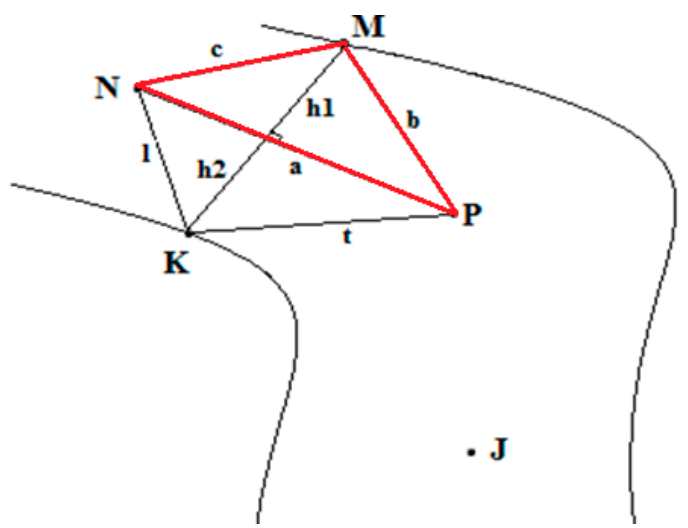

(b)

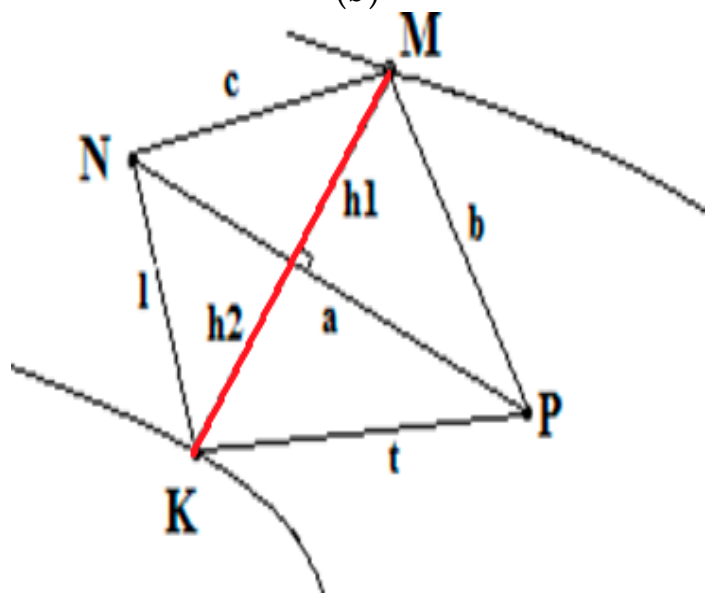

(d)

Figure 6. Technique used for the determination of the blood vessel diameters: (a) Presentation of the basic elements used (the two black arrows represents the Contour points and the three other black arrows represents the characteristic points); (b) Find the perimeter of a triangle; (c) Calculate the area of a triangle; (d) Calculate the heights of the triangles and the blood vessel diameters.

Step 1: Connect the first characteristic point $(N)$ and the second characteristic point $(P)$ in the same blood vessel branch to a straight line $\overline{N P}$. Then, we connect these two characteristic points at any chosen contour point that belongs to the same blood vessel branch $(\overline{N M}$ and $\overline{M P})$ (see Figure 6a).

Step 2: We calculate the Euclidian distance of the three lines with the following equations:

$$
\begin{aligned}
& \mathrm{a}=\sqrt{\left(x_{\mathrm{N}}-x_{\mathrm{P}}\right)^{2}+\left(y_{\mathrm{N}}-y_{\mathrm{P}}\right)^{2}} \\
& \mathrm{~b}=\sqrt{\left(x_{\mathrm{M}}-x_{\mathrm{P}}\right)^{2}+\left(y_{\mathrm{M}}-y_{\mathrm{P}}\right)^{2}} \\
& \mathrm{c}=\sqrt{\left(x_{\mathrm{N}}-x_{\mathrm{M}}\right)^{2}+\left(y_{\mathrm{N}}-y_{\mathrm{M}}\right)^{2}}
\end{aligned}
$$

Step 3: We calculate the heights of the different triangles MNP $(h 1)$ and KPP $(h 2)$, and the sum of these two heights in order to get the diameter of the blood vessel (see Figure $6 \mathrm{~d}$ ):

We can use Heron's formula to find the area of a triangle using the three side lengths:

$M, N$, and $P$, the three vertices of the triangle, and $a, b$, and $c$ the three sides respectively opposite to the three vertices. Then, we set $S=\frac{a+b+c}{2}$ (half perimeter) (see Figure 6b).

There is a formula (Equation (5)) to calculate the area of triangle (called A) (see Figure 6c):

$$
A=\sqrt{S(S-a)(S-b)(S-c)}
$$


Also, we can note that the area is given by the following formula (Equation (6)):

$$
\mathrm{A}=\frac{\mathrm{h} \times \mathrm{a}}{2}
$$

Then, we can deduce:

$$
\sqrt{S(S-a)(S-b)(S-c)}=\frac{h_{i} \times a}{2} \text { With } i=1,2 .
$$

From where:

$$
\mathrm{h}_{\mathrm{i}}=\frac{2 \times \mathrm{A}}{\mathrm{a}}
$$

\section{Results}

The system was implemented on a personal computer (PC) with an Intel Pentium B960 CPU @ $2.20 \mathrm{GHz}$ and $4 \mathrm{~GB}$ of RAM. The program was implemented in MATLAB ${ }^{\circledR} 2014 \mathrm{~b}$ and the results were also graphed with MATLAB ${ }^{\circledR}$.

\subsection{Segmentation of the Human Retina Image}

The determination of the blood vessel diameter using image analysis necessitates the initial segmentation of the retinal image so as to produce binary images. The typical path of making a binary image (Figure 7a) from a greyscale image (Figure $2 b$ ) is made by the thresholding method. Subsequently, the skeleton of the blood vessels is produced from the input binary image by the thinning process. This is shown in Figure $7 \mathrm{~b}$.

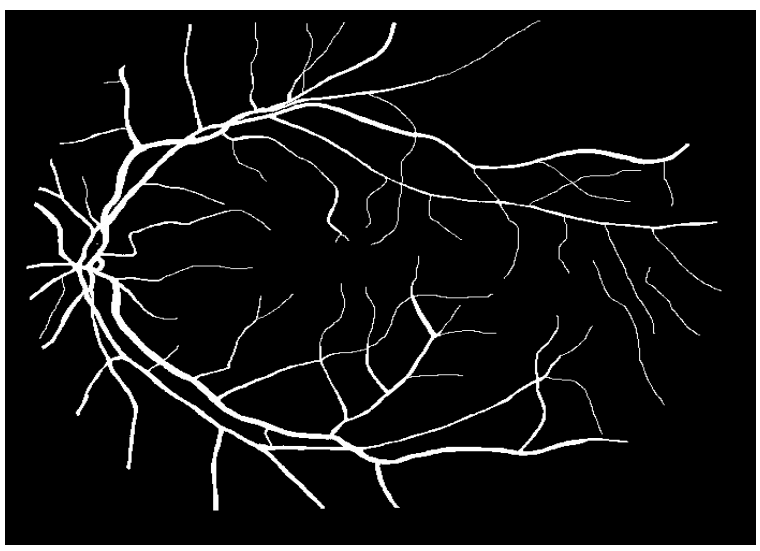

(a)

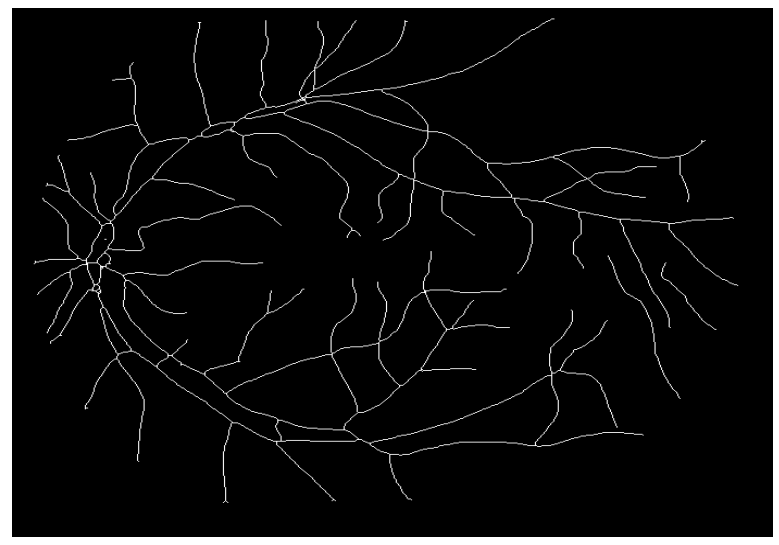

(b)

Figure 7. Binarization and skeletonization image: (a) Binary image; (b) Skeletonization image.

\subsection{Douglas-Peucker Algorithm}

To run the Douglas-Peucker algorithm, we began by determining the trajectory curve of each vessel. To accomplish this goal, we tried to detect the endpoints (Figure 8a), the bifurcation points (Figure $8 \mathrm{~b}$ ), and the internal points in the retinal image. 


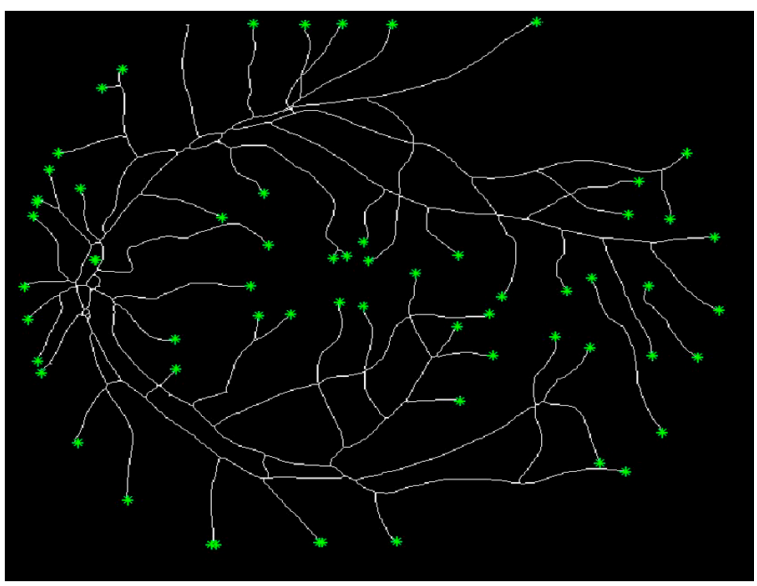

(a)

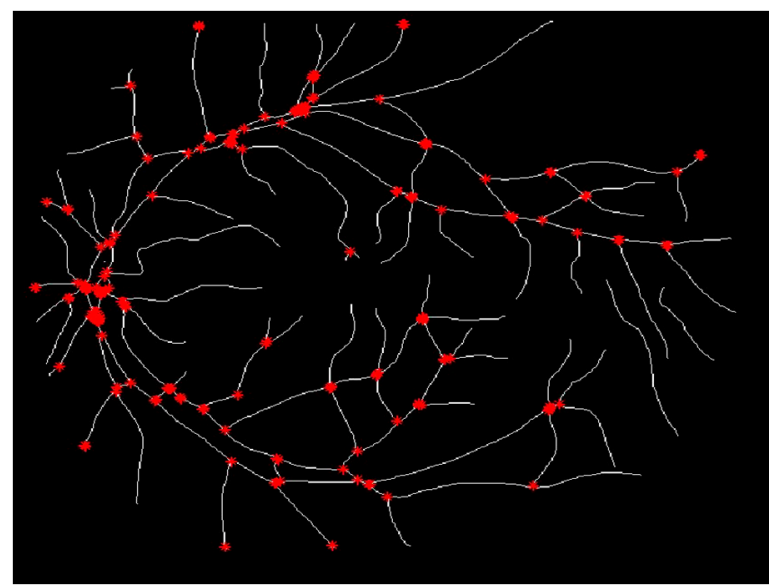

(b)

Figure 8. Detection of the endpoints and bifurcation points: (a) Endpoints; (b) Bifurcation points.

The Douglas-Peucker algorithm reduces the data storage amount and the number of points from a given line, while maintaining its principal geometrical and topological properties. The data compression ratio (DCR) (Figure 9) is defined as follows:

$$
D C R=\frac{(N-n)}{n} \mathbf{v}
$$

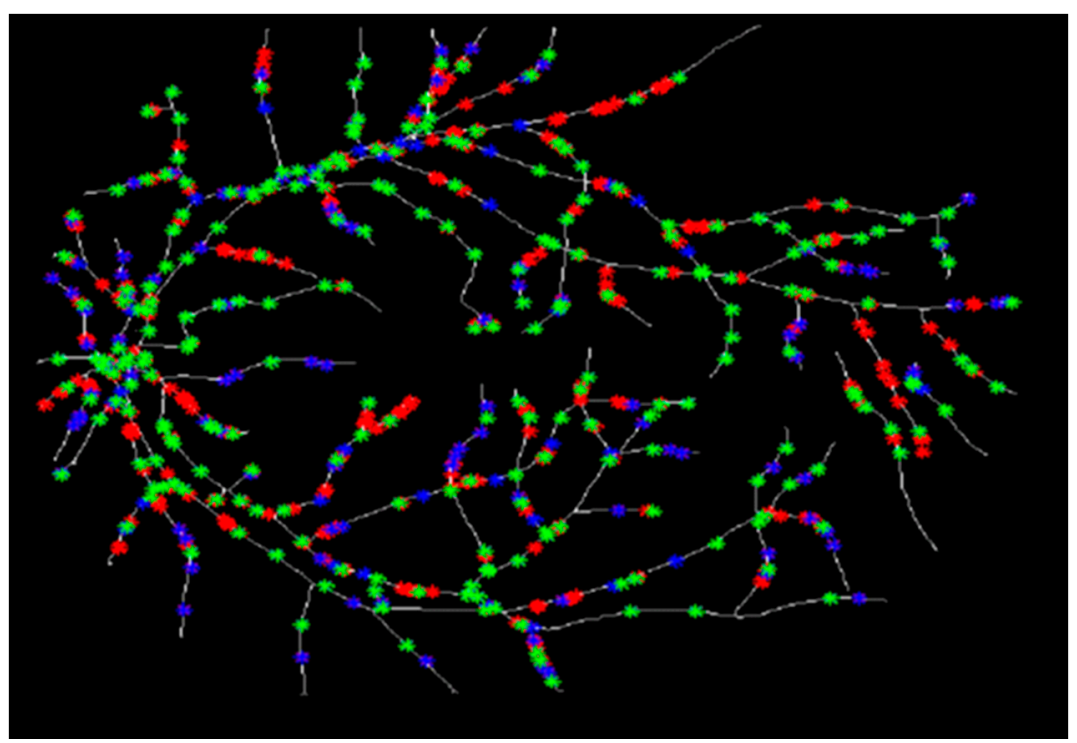

Figure 9. Determination of the characteristic points for three various values of $\varepsilon$. The red points show the characteristic points for $\varepsilon=0.5$, the blue points depict the characteristic points for $\varepsilon=1$, and the green points present the characteristic points for $\varepsilon=1$.

Figure 10a shows the number of characteristic points obtained for different tolerances values $\varepsilon$. We notice from this figure that the characteristic point number is between 1545 for $\varepsilon=2$ and 517 for $\varepsilon=0.5$, and Figure 10b shows that the compression ratio is around $92 \%$ for $\varepsilon=0.5$, and it can be improved to $96.6 \%$ for $\varepsilon=2$. It should be observed that the compression ratio and the characteristic point number vary according to the value of $\varepsilon$. If $\varepsilon$ is high, the number points detected is low, and vice versa. 


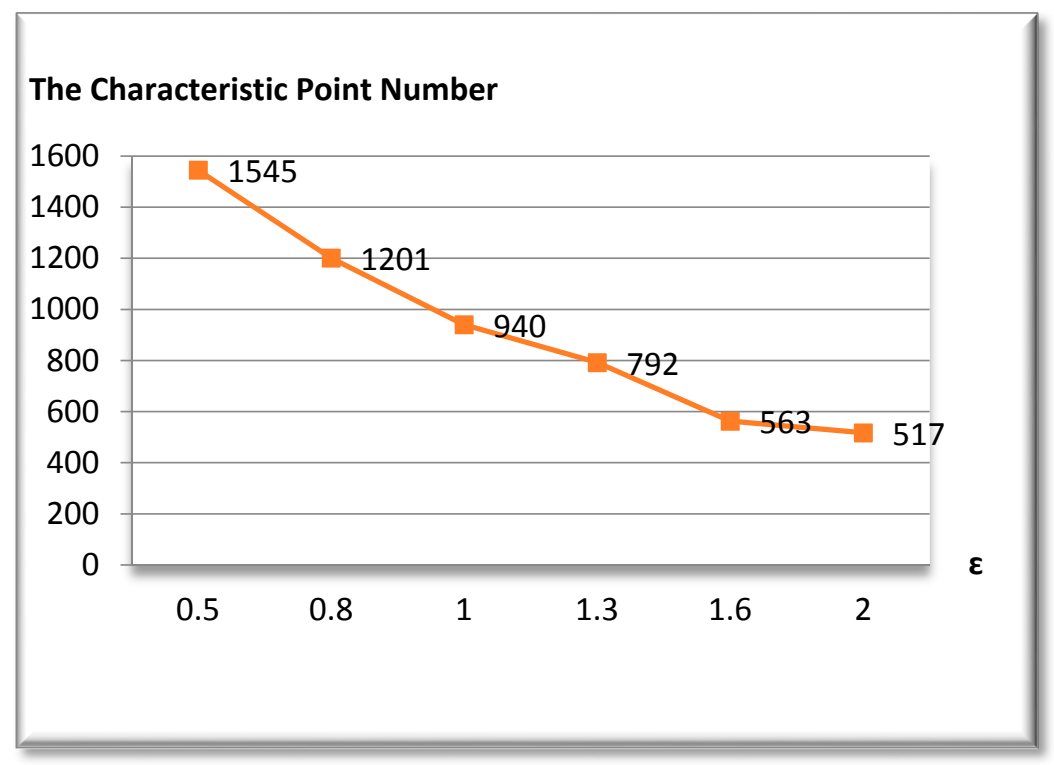

(a)

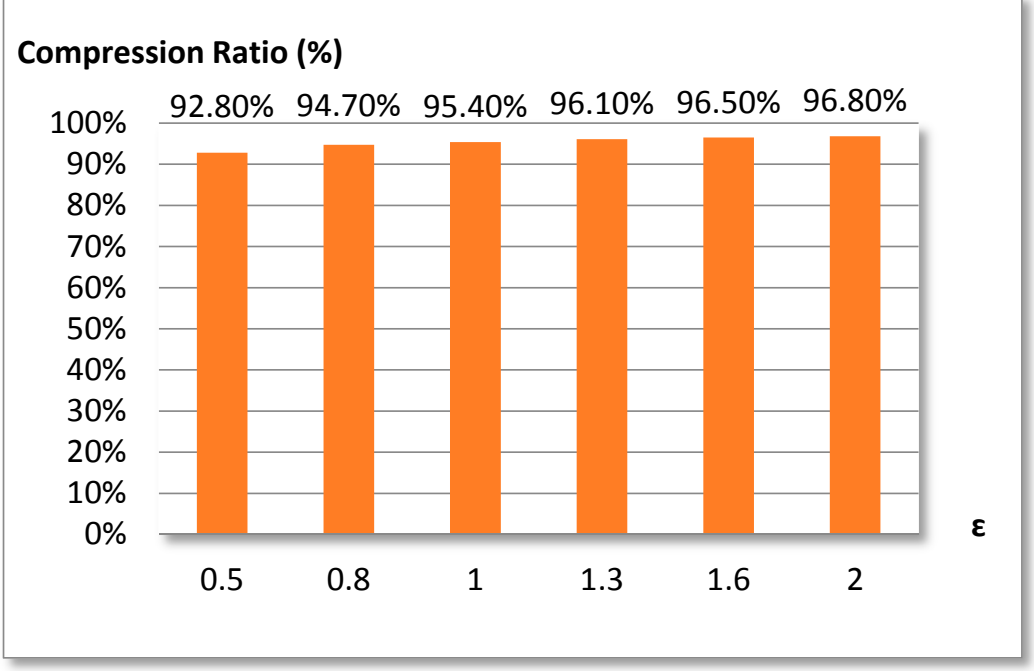

(b)

Figure 10. (a) The characteristic point number; (b) The compression ratio of the vascular tree.

\subsection{Diameter Measurement}

Figure 11a shows an example of contour detection by the active contours method. Figure 11b shows the characteristic point determination and represents the method used to calculate the blood vessel diameter.

To accomplish the goal of this research project, two databases were chosen in order to ensure the performance of the proposed algorithm. Three images were used for each database, the STARE database which is illustrated in Table 1 and the HRF database, which is shown in Table 2. 


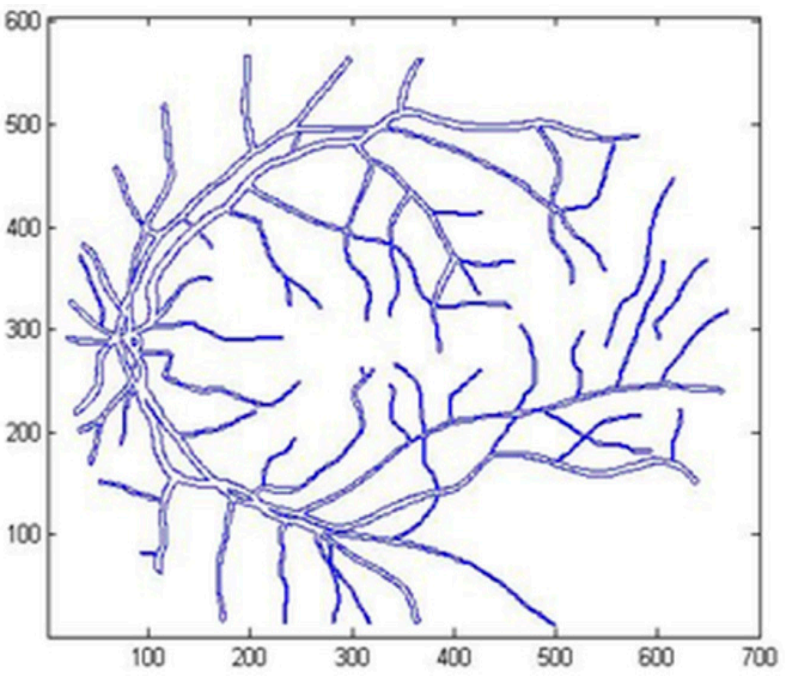

(a)

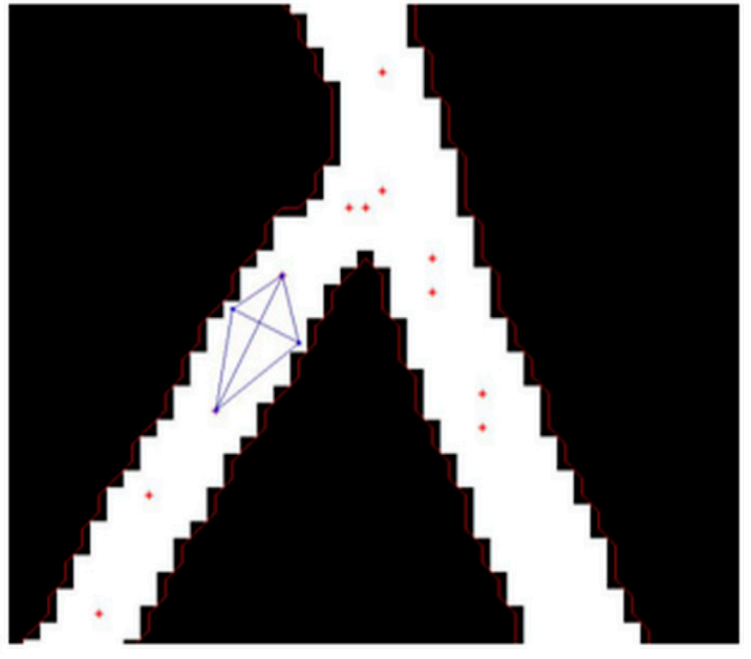

(b)

Figure 11. Calculation of the blood vessel diameter: (a) Contour detection; (b) Image depicting the calculation of the blood vessel diameter (the blue lines represents the triangles detection and the red dots represents the characteristic points).

The appropriate method to test and compare our measurement technique is to compare the same blood vessel measurements. To this end, we tested the accuracy of the algorithm presented using 30 widths obtained from six fundus images. Thus, in order to evaluate the effectiveness of our proposed program and also to obtain a more reliable analysis of the experimental data, we calculated the mean and standard deviation (SD) $[47,48]$ difference between the manual measurement and the measurement using the proposed method and noted here $E_{\mathrm{avg}}$ and SD respectively, as shown in the two tables below.

The standard deviation (SD) estimate is defined by the following formula $[47,48]$ :

$$
S D=\sqrt{\frac{\sum|x-\bar{x}|^{2}}{n}}
$$

The mean value $\left(E_{\mathrm{avg}}\right)$ is given by the following equation [47]:

$$
\operatorname{Eavg}=\frac{\sum|x-\bar{x}|}{n}
$$

where:

- $\quad x$ is the manual measurement value.

- $\quad \bar{x}$ is the measured value obtained using the proposed method.

- $\quad n$ is the number of test measurements. 
Table 1. Comparison of the diameter measurements (in pixels) between the proposed method and manual method for the STARE Database.

\begin{tabular}{|c|c|c|c|c|c|c|}
\hline Image & Vessel No & $\begin{array}{l}\text { Diameter } \\
\text { (Manual) }\end{array}$ & $\begin{array}{c}\text { Diameter } \\
\text { (Proposed Method) }\end{array}$ & Difference & $\begin{array}{c}\text { Standard } \\
\text { Deviation (SD) }\end{array}$ & $\begin{array}{c}\text { Average } \\
\left(E_{\text {avg }}\right)\end{array}$ \\
\hline \multirow{5}{*}{ im0002.ah.jpg } & 1 & 6.6212 & 6.6436 & -0.0224 & \multirow{5}{*}{0.0162} & \multirow{5}{*}{0.0211} \\
\hline & 2 & 5.3101 & 5.3099 & 0.0002 & & \\
\hline & 3 & 4.4721 & 4.4721 & 0 & & \\
\hline & 4 & 9.2540 & 9.2487 & 0.0053 & & \\
\hline & 5 & 13.6255 & 13.7002 & -0.0777 & & \\
\hline \multirow{5}{*}{ im0139.ah.jpg } & 1 & 4.0000 & 3.752 & 0.2480 & \multirow{5}{*}{0.1094} & \multirow{5}{*}{0.2390} \\
\hline & 2 & 4.1231 & 4.3872 & -0.2641 & & \\
\hline & 3 & 5.8310 & 5.642 & 0.189 & & \\
\hline & 4 & 8.0000 & 8.176 & -0.176 & & \\
\hline & 5 & 3.0000 & 2.682 & 0.318 & & \\
\hline \multirow{5}{*}{ im0077.ah.jpg } & 1 & 7.8102 & 7.9394 & -0.1292 & \multirow{5}{*}{0.1401} & \multirow{5}{*}{0.2752} \\
\hline & 2 & 4.2426 & 4.4792 & -0.2366 & & \\
\hline & 3 & 2.8284 & 2.674 & 0.1544 & & \\
\hline & 4 & 3.1623 & 2.6152 & 0.5471 & & \\
\hline & 5 & 8.9443 & 9.2528 & -0.3085 & & \\
\hline
\end{tabular}

Table 2. Comparison of the diameter measurements (in pixels) between the proposed method and manual method for the High-Resolution Fundus (HRF) Image Database.

\begin{tabular}{|c|c|c|c|c|c|c|}
\hline Image & Vessel No & $\begin{array}{l}\text { Diameter } \\
\text { (Manual) }\end{array}$ & $\begin{array}{c}\text { Diameter } \\
\text { (Proposed Method) }\end{array}$ & Difference & $\begin{array}{c}\text { Standard } \\
\text { Deviation (SD) }\end{array}$ & $\begin{array}{c}\text { Average } \\
\left(E_{\text {avg }}\right)\end{array}$ \\
\hline \multirow{5}{*}{ 10_h.tif } & 1 & 18.3848 & 18.3564 & 0.0284 & \multirow{5}{*}{0.0234} & \multirow{5}{*}{0.0307} \\
\hline & 2 & 5.000 & 4.887 & 0.1130 & & \\
\hline & 3 & 10.0499 & 10.0535 & -0.0036 & & \\
\hline & 4 & 2.8284 & 2.8301 & -0.0017 & & \\
\hline & 5 & 2.5381 & 2.5312 & 0.0069 & & \\
\hline \multirow{5}{*}{ 02_h.tif } & 1 & 3.0000 & 2.9654 & 0.0346 & \multirow{5}{*}{0.0092} & \multirow{5}{*}{0.0161} \\
\hline & 2 & 15.0000 & 15.028 & -0.0280 & & \\
\hline & 3 & 28.4253 & 28.4198 & 0.0055 & & \\
\hline & 4 & 20.0000 & 20.0035 & -0.0035 & & \\
\hline & 5 & 9.2195 & 9.2105 & 0.0090 & & \\
\hline \multirow{5}{*}{ 15_h.tif } & 1 & 22.6274 & 22.6248 & 0.0026 & \multirow{5}{*}{0.0063} & \multirow{5}{*}{0.0090} \\
\hline & 2 & 12.0000 & 12.0056 & -0.0056 & & \\
\hline & 3 & 21.0950 & 21.1256 & -0.0306 & & \\
\hline & 4 & 5.8310 & 5.8296 & 0.0014 & & \\
\hline & 5 & 7.6158 & 7.6205 & -0.0047 & & \\
\hline
\end{tabular}

Tables 1 and 2 provide a comparison of the accuracy between the manual measurements and the proposed method. This comparison is made respectively to the STARE Database [23] and High-Resolution Fundus (HRF) Image Database [24].

Column 5 in Tables 1 and 2 shows the difference between the manual measurements and the diameter measurements obtained by the proposed method. Columns 6 and 7 are the standard deviation $(\mathrm{SD})$ and mean $\left(E_{\mathrm{avg}}\right)$ of the difference between the types of measurements, respectively. According to both Tables 1 and 2, among the two datasets, the proposed method obtained the highest performance on the images from the High-Resolution Fundus (HRF) Image Database with the mean difference between 0.009 and 0.03 and standard deviation between 0.0063 and 0.0234 . The test using images from the STARE Database resulted in mean and standard deviation differences that were between 0.0211 and 0.2752 and 0.0162 and 0.1401 , respectively_these results are slightly higher than the results mentioned above. 


\section{Discussion}

The numerical results obtained by this study are compared with the values resulting from the formulations available in the literature.

Kumar et al. [11] tested their method on four sets of data, and their proposed method had the following performance: the Kick-Point Image Set (KPIS)database resulted in mean and SD difference values of 0.50 and 0.60 , respectively; the central light reflex validation test using the High Resolution Image Set (HRIS) database resulted in mean and SD difference values of -0.55 and 1.79 , respectively; the test using the Central Light Reflex Image Set (CLRIS) database result in mean and SD difference values of 0.21 and 0.79 , respectively; and tests using noisy pathological images (Vascular Disease Image Set (VDIS)dataset) resulted in mean and SD difference values of -0.64 and 1.18 , respectively. In Lowell et al. [17], the standard deviation difference generated by his method ranged between 0.124 and 0.464 pixels. On the other hand, Zhou et al. [19] reported that their approach based on the Gaussian 1-D model, had a standard deviation (SD) value of the order of 0.58. Add to that, the method of Chapman et al. [18] resulted in a mean difference between manually measured diameters and their test method in red-free images of about 3 pixels, and a standard deviation of 1.40 pixels. Gao et al. [15] provided blood vessel diameter measurements from a paired fluoresce in a 259 red-free image. The error generated by this technique was -0.9 for the mean difference value and 1.6 for the $\mathrm{SD}$ difference value, the error in this case is even larger. However, our results show that the maximum obtained difference does not exceed 0.3085 in absolute value. Moreover, the results we obtained are very consistent, with a small difference between the proposed method and manual method. Our technique has a standard deviation (SD) difference ranging between 0.0092 and 0.1401 pixels and produced a smaller mean difference value between 0.0090 and 0.2752 pixels.

We can notice that the presented algorithm is five times more accurate than the techniques proposed by Kumar et al. [11], and three times more precise than the method of Lowell et al. [17] and Zhou et al. [19]. We can also add, the obtained results from 30 measurement samples show that the proposed algorithm is over $70 \%$ more accurate than the other techniques.

Note that our proposed method provides information on the blood vessels in the most reliable and reproducible manner, and that it can be used to automatically diagnose the retinal blood vessels.

Another advantage is that the developed system requires less time because it has an execution time that is less than $2 \mathrm{~s}$. In addition, the results also show that this method appears to demonstrate good accuracy for all the databases.

The importance of this work lies in the fact that it can determine the morphological changes that target the human retinal blood vessels, and subsequently, it helps to facilitate several tasks such as diagnosing diseases during a clinical examination of the human retinal image and monitoring progress in vessels.

\section{Conclusions}

This objective of this study was mainly centered upon creating an automated computer system for measuring the blood vessel diameters of the human retina from an ocular fundus image. Several algorithms were used, including those for converting the image to a binary image to facilitate the determination of the bifurcations location, the endpoints, and the inner point to detect the vessel blood curves. Then, we determined the blood vessel contour using the active contour method. The next step which is described in this work was the determination of characteristic points by the Douglas-Peucker algorithm.

The final algorithm was used to calculate the diameter of the vessel. We presented a set of comparisons related to the vessel width between a manual measurement and the proposed computerized system. The obtained results are three times more precise than the other methods and over $70 \%$ more accurate than the other techniques. It can be noted that our developed system requires less time and provides more reliable and reproducible information regarding the morphology of blood vessels. 
Author Contributions: Guedri Hichem and Mariem Ben Abdallah performed the literature search for this review, Fraj Echouchene and Hafedh Belmabrouk drafted the initial manuscript.

Conflicts of Interest: The authors declare no conflict of interest.

\section{References}

1. Vidal, F.P.; Bello, F.; Brodlie, K.W.; John, N.W.; Gould, D.; Phillips, R.; Avis, N.J. Principles and applications of computer graphics in medicine. Comput. Graph. Fourm 2006, 25, 113-137. [CrossRef]

2. Wernick, M.N.; Bouman, C.A.; Leahy, R.M.; Duncan, J.S. The Roles of Signal Processing in Medical Imaging. IEEE Signal Process. Mag. 2010, 27, 12-140. [CrossRef]

3. Quistgaard, J. Signal Acquisition and Processing in Medical Diagnostic Ultrasound. IEEE Signal Process. Mag. 1997, 14, 67-74. [CrossRef]

4. McCloy, R.; Stone, R. Virtual reality in surgery. Br. Med. J. 2001, 323, 912-915. [CrossRef]

5. Evans, A.C.; Marret, S.; Collins, L.; Peters, T.M. Anatomical functional correlative analysis of the human brain using 3d imaging systems. Med. Imaging III Image Process. 1989, 1092, 264-275.

6. Nguyen, T.T.; Wang, J.; Islam, F.; Mitchell, P.; Tapp, R.J.; Zimmet, P.Z.; Simpson, R.; Shaw, J.; Wong, T.Y. Retinal Arteriolar Narrowing Predicts Incidence of Diabetes: The Australian Diabetes, Obesity and Lifestyle (AusDiab) Study. Diabetes 2008, 57, 536-539. [CrossRef] [PubMed]

7. Wong, T.Y.; Klein, R.; Sharrett, A.R.; Schmidt, M.I.; Pankow, J.S.; Couper, D.J.; Klein, B.E.; Hubbard, L.D.; Duncan, B.B.; ARIC Investigators. Retinal arteriolar narrowing and risk of diabetes mellitus in middle-aged persons. J. Am. Med. Assoc. 2002, 57, 2528-2533. [CrossRef]

8. Li, H.; Hsu, W.; Lee, M.L.; Wang, H. A piecewise Gaussian model for profiling and differentiating retinal vessels. In Proceedings of the International Conference on Image Processing (ICIP '03), Catalonia, Spain, 14-18 September 2003; Volume 1, pp. 1069-1072.

9. Xu, X.; Reinhardt, J.M.; Hu, Q.; Bakall, B.; Tlucek, P.S.; Bertelsen, G.; Abràmoff, D.M. Retinal Vessel Width Measurement at Branchings Using an Improved Electric Field Theory-Based Graph Approach. PLoS ONE 2012, 7, e49668. [CrossRef] [PubMed]

10. Bankhead, P.; Scholfield, C.N.; McGeown, J.G.; Curtis, T.M. Fast Retinal Vessel Detection and Measurement Using Wavelets and Edge Location Refinement. PLoS ONE 2012, 7, e32435. [CrossRef] [PubMed]

11. Kumar, D.K.; Aliahmad, B.; Hao, H. Retinal vessel diameter measurement using unsupervised linear discriminant analysis. ISRN Ophthalmol. 2012, 2012, 1-7. [CrossRef] [PubMed]

12. Bhuiyan, A.; Nath, B.; Chua, J.; Kotagiri, R. Vessel cross-sectional diameter measurement on color retinal image. In Proceedings of the International Joint Conference, BIOSTEC 2008 Funchal, Madeira, Portugal, 28-31 January 2008; pp. 214-227.

13. El Abbadi, N.; Al Saadi, E. Bood vessel diameter measurement on retinal image. J. Comput. Sci. 2014, 10, 879-883. [CrossRef]

14. Gao, X.W.; Bharath, A.; Stanton, A.; Hughes, A.; Chapman, N.; Thom, S. Measurement of vessel diameters on retinal for cardiovascular studies. In Proceedings of the Medical Image Understanding and Analysis, Birmingham, UK, 16-17 July 2001; pp. 1-4.

15. Gao, X.W.; Bharath, A.; Stanton, A.; Hughes, A.; Chapman, N.; Thom, S. Quantification and characterisation of arteries in retinal images. Comput. Methods Programs Biomed. 2000, 63, 133-146. [CrossRef]

16. Tizon, A.; Courtney, J. Blood Vessel Diameter Estimation System Using Active Contours. In Proceedings of the 2011 Irish Machine Vision and Image, Dublin, Ireland, 8-9 September 2011.

17. Lowell, J.; Hunter, A.; Steel, D.; Basu, A.; Ryder, R.; Kennedy, R.L. Measurement of Retinal Vessel Widths From Fundus Images Based on 2-D Modeling. IEEE Trans. Med. Imaging 2004, 23, 1196-1204. [CrossRef] [PubMed]

18. Chapman, N.; Witt, N.; Gao, X.; Bharath, A.; Stanton, A.; Thom, S.; Hughes, A. Computer algorithms for the automated measurement of retinal arteriolar diameters. Br. J. Ophthalmol. 2001, 85, 74-79. [CrossRef] [PubMed]

19. Zhou, L.; Rzeszotarski, M.S.; Singerman, L.J.; Chokreff, J.M. The detection and quantification of retinopathy using digital angiograms. IEEE Trans. Med. Imaging 1994, 13, 619-626. [CrossRef] [PubMed] 
20. Gang, L.; Chutatape, O.; Krishnan, S.M. Detection and measurement of retinal vessels in fundus images using amplitude modified second-order Gaussian filter. IEEE Trans. Biomed. Eng. 2002, 49, 168-172. [CrossRef] [PubMed]

21. Zhao, X.; Duan, W.; Lin, T.; Li, B. A method of retinal vessel width measurement. In Proceedings of the 2nd International Conference on Computer and Automation Engineering (ICCAE'10) 2010, Singapore, 26-28 February 2010; pp. 443-446.

22. Aliahmad, B.; Kumar, D.K.; Janghorban, S.; Azemin, M.Z.C.; Hao, H.; Kawasaki, R. Retinal vessel diameter measurement using multi-step regression method. In Proceedings of the Biosignals and Biorobotics Conference (BRC '12), Manaus, Brazil, 9-11 January 2012; pp. 1-4.

23. STARE (STructured Analysis of the Retina) Project Website. Available online: http://www.ces.clemson.edu/ ahoover/stare (accessed on 10 December 2016).

24. High-Resolution Fundus (HRF) Image Database Website. Available online: http://www5.cs.fau.de/ research/data/fundus-images (accessed on 15 December 2016).

25. Hoover, A.; Kouznetsova, V.; Goldbaum, M. Locating Blood Vessels in Retinal Images by Piece-wise Threhsold Probing of a Matched Filter Response. IEEE Trans. Med. Imaging 2000, 19, 203-210. [CrossRef] [PubMed]

26. Otsu, N. A Threshold Selection Method from Gray-Level Histograms. IEEE Trans. Syst. Man Cybern. 1979, 9, 62-66. [CrossRef]

27. Greig, D.M.; Porteous, B.T.; Seheult, A.H. Exact maximum a posteriori estimation for binary images. J. $R$. Stat. Soc. Ser. B 1989, 51, 271-279.

28. Akram, M.U.; Tariq, A.; Khan, S.A. Retinal image blood vessel segmentation. In Proceedings of the International Conference on Information and Communication Technologies, ICICT'09, Karachi, Pakistan, 15-16 August 2009; pp. 181-192.

29. El Abbadi, N.K.; Al Saadi, E.H. Blood vessels extraction using mathematical morphology. J. Comput. Sci. 2013, 9, 1389-1395. [CrossRef]

30. Haralick, R.M.; Shapiro, L.G. Computer and Robot Vision; Addison-Wesley: Boston, MA, USA, $1992 ;$ Volume 1.

31. Kong, T.Y.; Rosenfeld, A. Topological Algorithms for Digital Image Processing; Elsevier Science, Inc.: North Holland, The Netherlands, 1996.

32. Lam, L.; Seong-Whan, L.; Ching, Y.S. Thinning Methodologies-A Comprehensive Survey. IEEE Trans. Pattern Anal. Mach. Intell. 1992, 14, 869-885. [CrossRef]

33. Bouix, S.; Siddiqi, K.; Tannenbaum, A. Flux driven automatic centerline extraction. Med. Image Anal. 2005, 14, 209-221. [CrossRef] [PubMed]

34. Dikici, E.; O'Donnell, T.; Grady, L.; White, R. Coronary Artery Centerline Tracking Using Axial Symmetries. Available online: http://www.midasjournal.org/browse/publication/586 (accessed on 1 December 2016).

35. Zhou, Y.; Toga, A.W. Efficient Skeletonization of Volumetric Objects. IEEE Trans. Vis. Comput. Graph. 1999, 5 , 196-209. [CrossRef] [PubMed]

36. Zhao, F.; Tang, X. Preprocessing and postprocessing for skeleton-based fingerprint minutiae extraction. Pattern Recognit. 2007, 40, 1270-1281. [CrossRef]

37. Guedri, H.; Malek, J.; Belmabrouk, H. Reconstruction of the human retinal blood vessel by fractal interpolation. J. Theor. Appl. Inf. Technol. 2016, 83, 227-233.

38. Guedri, H.; Chouchene, F.; Belmabrouk, H. 3D Model Reconstruction of Blood Vessels in The Retina with Tubular Structure. Int. J. Electr. Eng. Inform. 2015, 7, 724-734.

39. White, E.R. Assessment of line-generalization algorithms using characteristic points. Am. Cartogr. 1985, 12, 17-28. [CrossRef]

40. Marino, J.S. Identification of characteristic points along naturally occurring lines. Cartogr. Int. J. Geogr. Inf. Geovisualization 1979, 16, 70-80. [CrossRef]

41. Douglas, D.H.; Peucker, T.K. Algorithms for the reduction of the number of points required to represent a line or its caricature. Can. Cartogr. 1973, 10, 112-122. [CrossRef]

42. Hershberger, J.; Snoeyink, J. Speeding up the Douglas-Peucker line-simplification algorithm. In Proceedings of the Fifth International Symposium on Spatial Data Handling, Charleston, SC, USA, 3-7 August 1992; Volume 1, pp. 134-143.

43. Kass, M.; Witkin, A.; Terzopoulos, D. Snakes: Active Contour Models. Int. J. Comput. Vis. 1988, 1, 321-331. [CrossRef] 
44. Caselles, V. Geodesic Active Contours. Int. J. Comput. Vis. 1997, 22, 61-79. [CrossRef]

45. Tajik, H.R.; Rahebi, J. Diffuse Objects extraction in Coronal Holes Using Active Contour Means Model. ACSIJ Adv. Comput. Sci. 2013, 2, 55-61.

46. Derraz, F.; Beladgham, M.; Khelif, M. Application of active contour models in medical image segmentation. In Proceedings of the Information Technology: Coding and Computing, Las Vegas, NV, USA, 5-7 April 2004; Volume 2, pp. 675-681.

47. Webster, C.S.; Merry, A.F. The standard deviation and the standard error of the mean. Anaesthesia 1997, 52, 183. [PubMed]

48. Altman, D.G.; Bland, J.M. Standard deviations and standard errors. BMJ 2005, 52331. [CrossRef] [PubMed]

(C) 2017 by the authors. Licensee MDPI, Basel, Switzerland. This article is an open access article distributed under the terms and conditions of the Creative Commons Attribution (CC BY) license (http:/ / creativecommons.org/licenses/by/4.0/). 